\title{
Guest editorial: special issue on network and systems support for games
}

\author{
Maha Abdallah $\cdot$ Khaled Boussetta $\cdot$ \\ Wei Tsang Ooi
}

Published online: 11 July 2014

(c) Springer-Verlag Berlin Heidelberg 2014

A recent market forecast has predicted that the global market
for video games will rise to $\$ 82$ billion in 2017 (http://www.
forbes.com/sites/johngaudiosi/2012/07/18/new-reports-
forecasts-global-video-game-industry-will-reach- 82 -bil
lion-by-2017/). The growth is driven by factors including
advances in graphics hardware and proliferation of mobile
devices. The ubiquity of network connections has also
driven up the demand for online gaming, with the market
forecasted to reach $\$ 35$ billion by 2017 (http://www.for
bes.com/sites/johngaudiosi/2012/07/18/new-reports-fore
casts-global-video-game-industry-will-reach- 82 -billion-
by-2017/). This increasing number of game players desire
better playing experiences, straining the underlying net-
work and system architecture, and introduces new research
questions that require synthesis of a variety of research
areas.

This special issue focuses on network and systems aspects of games, and covers research and engineering topics that help understand networked games of today and enable the next generation of future networked games. We invited 19 selected papers published in the NetGames 2012

\section{Abdallah ( $\square)$}

Laboratoire d'Informatique de Paris 6 (LIP6), Pierre and Marie Curie University, 4, Place Jussieu, 75005 Paris, France e-mail: Maha.Abdallah@lip6.fr

\section{K. Boussetta}

Laboratoire de Traitement et Transport de l'Information (L2TI), University Paris 13, 99 Avenue Jean-Baptiste Clément, 93430 Villetaneuse, France

e-mail: Khaled.boussetta@univ-paris13.fr

W. T. Ooi

Department of Computer Science, National University of Singapore, Singapore 117417, Singapore

e-mail: ooiwt@comp.nus.edu.sg
Workshop to expand their work and to submit to the special issue. We also received seven papers that are submitted as a result of the open call for papers. In total, 18 papers have been submitted. After a rigorous reviewing process, we accepted 11 of the submissions to appear in this special issue. The accepted papers cover a wide range of topics in network and systems support for games, including cloud gaming, mobile gaming, and security.

Cloud gaming has recently emerged as a new service that offers the possibility to play online games using any terminal, such as a tablet, a smartphone, or a TV screen. The concept is based on cloud computing principles, where most of the processing is achieved in the cloud and the audio/video rendering is streamed to thin terminals. Pushing the complexity toward data centers and network infrastructures offers many advantages to the customers and to the gaming industry, but it also raises many technical and scientific challenges that have recently attracted a growing attention from the researchers and the developers. Addressing these issues requires, as a preliminary step, a better understanding of this emerging service.

The first five papers of this special issue address a wide range of important issues related to cloud gaming. The Paper "Dissecting the Protocol and network Traffic of the OnLive Cloud Gaming platform" provides a detailed analysis of the traffic associated to a popular cloud gaming platform. The different phases of the game sessions are identified and the associated data traffic is characterized in terms of bandwidth, packets size and arrival time distributions. Using these observations, the authors propose a very useful mathematical model of cloud gaming traffic.

The paper "On the Performance of OnLive Thin Client Games" aims to provide a deep understanding of the traffic associated with cloud gaming, where traffic features are studied and compared to other multimedia services like 
VoIP or video streaming. In particular, the authors provide a deep analysis of traffic behavior under the effect of different network imperfections, such as packets' loss, delays variation and bandwidth limitation, and discuss also the impact of these network's turbulences on the quality of experience.

Achieving a trade-off between bitrate reduction and the perceptual quality of streamed video games is the objective of the work presented in the paper "A Game Attention Model To Efficiently Allocate Bit Rate in cloud Gaming". The authors propose a video coding method that exploits some characteristics of the human visual system and uses high-level game-specific semantics to differentiate which areas must be coded with higher quality from those with lower quality. The subjective evaluation tests show that the method can result in $25 \%$ less bandwidth with little to no perceived impact on the gameplay.

In the paper "A Hybrid Edge-Cloud Architecture for Reducing On-Demand Gaming Latency", the authors investigate the feasibility of deploying video games in the cloud. Through a large-scale measurement study, the authors identify latency as a significant factor that affects the deployment of game applications in existing cloud infrastructures. Several alternative deployment models are then examined and compared, whereby the authors argue in favor of a hybrid model that consists of using edge-servers in addition to the cloud for ensuring low latency as well as high serving and hosting capacities. The study is completed by examining various edge-server site selection and game placement strategies, and their effect on the ability of the system to serve end-users.

The paper "SLA-based Operations of Massively Multiplayer Online Games in Clouds" proposes a new model for hosting and provisioning MMOGs that involve three components: game providers, game operators, and resource providers, with the game operators acting as intermediary that provisions resources intelligently for game providers. The authors showed that such three-tier model can mitigate the effects of resource failures and reduces the operation cost significantly.

With the current mobile computing revolution and the proliferation of smart devices, users are more likely to use smart mobile devices for a wide variety of applications, including video games. Unlike other applications, video games in general, and multiplayer online games in particular, are extremely latency-sensitive and resourceintensive in terms of the processing power, display capabilities, and network bandwidth (specifically in cloud gaming) that they require. As the mobile gaming trend will continue to grow, driven by strong user demand, significant challenges thus remain to be solved to bring realistic and immersive game experiences to resourceconstrained devices.
The next two papers address some of the current challenges facing the mobile gaming technology. In the paper "Opportunistic Mobile Games Using Public Transportation Systems: a Deployability Study", the authors studied how a location-based and context-based mobile games can be implemented over contact-based opportunistic networking provided by public transportation systems. The paper provides an insight into mobile game designers on how to design a game that requires contact among players in a complex urban environment.

The paper "Lightweight Graphics Instrumentation for Game-State specific Power Management in Android" tackles the problem of power consumption of mobile games running on resource-constrained devices. The authors propose a power saving approach that detects a game's current state and predicts future workload of frames to achieve state-specific power management by dynamically scaling the processor's frequency according to the target frame rate. By intercepting calls to the graphics library, the proposed approach cleverly sits outside the game and does not require the modification of the game's source code.

Security and trustworthiness are undoubtedly among the most challenging issues facing any fully distributed P2P application and service, and video games are no exception. Scalability, fault-tolerance, and cost-effectiveness are among the key motivations behind the numerous efforts underlying the design of fully distributed multiplayer online games. However, these advantages come at the high price of an architecture lacking a trusted authority, and thus more prone to cheating and other general security breaches. As such, security and trustworthiness issues need to be addressed in order to make fully distributed multiplayer games a viable option, which is the focus of the next two papers.

The paper "Towards a scalable refereeing system for online gaming" addresses the problem of cheating, central to fully decentralized multiplayer gaming architectures. A probabilistic peer-to-peer-based solution for refereeing online multiplayer games is proposed, where referees are chosen dynamically and in sufficient number to reduce cheating to an acceptable level, making peer-to-peer gaming architectures viable.

Securely trading virtual items in a completely distributed way in multiplayer games is the focus of the paper "Secure Peer-to-Peer Trading in Small and Large-Scale Multiplayer Games". Following a peer-to-peer approach, the authors propose a scalable and secure trading protocol where items are exchanged fairly and are not duplicated. Interestingly, the proposed protocol, discussed in the context of both small and large-scale multiplayer games, can be used for other applications beyond games where fairly trading digital assets is needed. 
While the papers above have investigated into various research issues, the next paper has tried to improve on the research methodologies of networked games research. To evaluate a solution in a reproducible way, researchers often resort to simulations based on synthetic workloads. While traditionally, such workloads are often generated based on a mobility model of players, the paper "On Synthetic Workloads for Multiplayer Online Games; A Methodology for Generating Representative Shooter Game Workloads" found that modeling the AI of the player can lead to a more representative workload that is closer to that generated by real human players, and therefore could lead to a more realistic synthetic workload for evaluation.

Last but not least, our final paper in this special issue, "QoE Assessment of Fairness in Networked Game with Olfaction: Influence of Time It Takes for Smell to Reach Player" ventures into future networked gaming experiences by including olfactory as one of the media involved in playing games. In particular, the paper studied the effect of network latency on the perceived fairness of a game where players need to make a decision based on the odor emitted during game play.

At the end, we would like to express our gratitude to all the reviewers of this special issue, as well as all the NetGames 2012 TPC members whose feedback and rigorous observations and comments directly influenced the high quality of the extended versions of the NetGames 2012 selected papers.

List of Reviewers:

- Nadjib Achir

- Toufik Ahmed

- Azeddine Beghdadi

- Anand Bhojan

- Jean Botev

- Surendar Chandra

- Kuan-Ta Chen

- Mark Claypool

- Magda El-Zarki

- Markus Esch

- Wu-chang Feng

- Wu-chi Feng

- Stefano Ferretti

- Chris GauthierDickey

- Steven Gianvecchio

- Carsten Griwodz

- Pal Halvorsen

- Mohamed Hefeeda

- Cheng-Hsin Hsu

- Chun-Ying Huang

- Polly Huang

- Alexandru Iosup

- Joaquin Keller
- Bettina Kemme

- Cristina Videira Lopes

- Dario Maggiorini

- Gustavo Marfia

- Ketan Mayer-Patel

- Niall Murray

- Ali Nazari

- Timo Ojala

- Claudio Palazzi

- Julien Ponge

- Cheryl Savery

- Gregor Schiele

- Shervin Shirmohammadi

- Gwendal Simon

- Hwee Xian Tan

- Arno Wacker

- Shinya Yamamoto

- Sebastian Zander

- Suiping Zhou

- Roger Zimmermann

Maha Abdallah is currently an Associate Professor at Pierre and Marie Curie University (UPMC). She received a Ph.D. degree (with highest Honors) in Computer Science from the University of Versailles in 2001. She then spent a year at the Department of Computer Science at the University of California, Santa Barbara (UCSB) as a research fellow before joining UPMC in 2002. Her current research interests are in distributed and cloud computing, covering distributed algorithms and systems, fault-tolerant systems, and scalable systems and networks, with current special focus on interactive 3D media applications, namely massively multiplayer virtual environments and online games. For more information, see: http://www-poleia.lip6.fr/ abdallah/.

Khaled Boussetta is an Associate Professor at Paris 13 University, since 2004. He received his Ph.D. (with highest Honors) in Computer Science from the University of Versailles, France in 2003. He then spent a year as a Postdoc at LIP6 laboratory at Pierre and Marie Curie University (UPMC) before joining Paris 13 University in 2004. Since September 2012, he joined the INRIA UrbaNet team located at CITI labs-INSA Lyon for a 2 years sabbatical. His research interests cover design, modeling, and performance evaluation of wireless networks and multimedia applications. For more information, see: http://www-12ti.univ-paris13.fr/ boussetta/.

Wei Tsang Ooi is an Associate Professor at the National University of Singapore (NUS). He received his B.Sc. (Hon.) degree from the Department of Information Systems and Computer Science (now School of Computing), NUS in 1996, and his Ph.D. in Computer Science from Cornell University, Ithaca, NY in 2001. He later spent a year as postdoc at Berkeley Multimedia Research Center at U.C. Berkeley before joining NUS in 2002. His research interests include multimedia, computer networking, mobile computing, and distributed systems. For more information, see: http://www.comp.nus.edu.sg/ $\sim$ ooiwt/. 